\title{
EVALUACIÓN DE OBJETIVOS DE CONSERVACIÓN DE ÁREAS PROTEGIDAS A PARTIR DEL ANÁLISIS DEL ÁREA DE CAMPEO Y POBLACIÓN MÍNIMA VIABLE DE ESPECIES DE FÉLIDOS Y CÁNIDOS. EL PARQUE NACIONAL TOROTORO (POTOSÍ, BOLIVIA) COMO EJEMPLO
}

\section{CONSERVATION OBJECTIVES OF PROTECTED AREA'S EVALUATION FROM THE ANALYSIS OF HOME RANGE AND MINIMUM VIABLE POPULATIONS OF FELIDAE AND CANIDAE SPECIES. TOROTORO NATIONAL PARK (POTOSI, BOLIVIA), AN EXAMPLE}

\author{
Enrique Richard ${ }^{1,2}$, Francisco Fontúrbel Rada ${ }^{3 *}$ y Gonzalo García Crispieri ${ }^{4}$
}

\section{Resumen}

Se analizó la relación superficie-perímetro actual del Parque Nacional Torotoro (Potosí, Bolivia), y en función a ella se calcularon los índices de superficie mínima indispensable para poblaciones de Puma concolor, Oreailurus jacobita y Lycalopex culpaeus; datos que, contrastados con la superficie efectiva de conservación del área protegida (47\% de la del total) muestran una clara deficiencia, puesto que la superficie mínima necesaria para conservar una población viable sería de 105 veces la correspondiente al área protegida $\left(166 \mathrm{~km}^{2}\right)$ para $P$. concolor, 273 veces para $O$. jacobita y 5 veces para $L$. culpaeus.

Palabras clave: Área Protegida, superficie y población mínima viable, área de campeo, metapoblación, fragmentación, corredor de biodiversidad

\begin{abstract}
The actual relation surface-perimeter of Torotoro National Park (Potosi, Bolivia) was analyzed, and on its basis, minimal required surface's indexes were calculated for Puma concolor, Oreailurus jacobita and Lycalopex culpaeus populations. Data obtained were compared to the current effective conservation surface ( $47 \%$ of the total surface ) and showed a clear deficiency, because the minimum surface required to conserve minimal viable populations of these carnivores is 105 times the Park's protected surface $\left(166 \mathrm{~km}^{2}\right)$ for P. concolor, 273 times for O. jacobita and 5 times for L. Culpaeus.
\end{abstract}

Keywords: Natural Protected Area, minimal viable population and surface, home range, metapopulation, breakness, biodiversity corridor

\section{Introducción y antecedentes}

El diseño de Áreas Naturales Protegidas (ANP) constituye una parte integral de la biología de la conservación (Barzetti, 1993; Moyle \& Sato, 1991; Meffe et al. 1999, Smith \& Smith 2001). Esta rama de la ciencia dirige sus esfuerzos a la prevención de extinciones y la protección de la biodiversidad.

En el diseño de APN convergen varias ciencias, entre ellas, la ecología, dinámica y genética de poblaciones y la biogeografía. Uno de sus principales aportes proviene de la teoría del equilibrio insular (Fernández, 2000; Smith \& Smith, 2001), de la cual emerge el supuesto que en relación a su entorno, las ANP se comportan como islas de ambiente natural en un mar de ambiente antropogénico y, por tanto, están sujetas a la dinámica que las caracteriza, desde el punto de vista de la genética de poblaciones, que se manifiesta en un incremento de riqueza de especies con el incremento de área, siguiendo una función exponencial (Hastings \& Harrison, 1994; Klug \& Cummings, 1999; Smith \& Smith 2001). Diversos autores (Diamond, 1975; Sullivan \& Shaffer, 1975; Meffe et al., 1999) han sugerido principios para el diseño de ANP maximizando la preservación de la biodiversidad a partir de las relaciones de superficie y forma de las mismas (Schoener, 1998). Algunas de las propuestas sugeridas y derivadas de esta hipótesis, donde los diseños circulares, unitarios y grandes son teóricamente mejores (Moyle \& Sato, 1991; Meffe et al., 1999). Otros autores proponen la utilización de los parámetros que mejor predicen la diversidad específica, los efectos de la fragmentación y el aislamiento sobre la diversidad de especies (Schwartz et al., 2000; Hector et al., 2001).

A todo esto, se debe agregar que la fragmentación de los hábitats naturales por acción antropógena ocurre a escalas breves de tiempo (en relación a escalas ecológicas y evolutivas) y con efectos más acentuados que los naturales (Forman \& Godron, 1986). En tal sentido, el aislamiento de un área por efecto antropógeno (por ejemplo, la creación de un ANP) deriva en la declinación del número de especies 
Diciembre 2006

por extinción o emigración pero, pero sin que se favorezca la inmigración a la misma (Pianka, 1982; Fernández, 2000; Thomas \& Kunin, 1999). Consecuentemente, cuanto más pequeña y aislada sea el ANP más susceptible será a la pérdida de biodiversidad.

Harrison (1991) propone cuatro modelos metapoblacionales: (1) La metapoblación “clásica” compuesta por un grupo de parches pequeños que están más predispuestos a la extinción mientras más pequeños son, pero que se encuentran lo suficientemente cerca como para permitir la recolonización, evitando procesos de degradación genética. (2) La metapoblación en "parches", compuesta de un grupo de parches pequeños que se encuentran lo suficientemente cerca como para funcionar como una población. (3) La metapoblación "continente-isla" compuesto de un parche grande (“continente”) y un número variable de parches pequeños ("islas”) suficientemente cercanos para recibir una migración continua de los individuos del parche grande y prevenir una extinción local, y (4) la metapoblación en "desequilibrio" compuesta de varios parches pequeños aislados que actúan independiente y por lo tanto, cada uno muestra una tendencia a la extinción. Los análisis de Harrison (1991) plantean que los modelos 2 y 3 son los más estables, mientras que el modelo 4 sería el resultado final de la fragmentación y pérdida de conectividad espacial entre los parches. Dichos criterios fueron aplicados por Acosta-Jamett et al. (2003) para el caso de Oncifelis guigna (Mammalia: Felidae) en bosques fragmentados de Chile central.

Otros dos factores a considerar como amenaza de la biodiversidad son: el efecto de borde (relación superficie-perímetro a favor de este último) y los efectos externos (fuego, contaminación, especies introducidas, etc.), de los cuales surge el concepto de zonas buffer o de amortiguamiento. Éstas, tienen por finalidad proveer un gradiente de condiciones que actúan como barreras a las perturbaciones externas. Además, las zonas buffer, deberían proveer beneficios socioeconómicos dentro del marco del desarrollo sustentable (Vides \& Korn, 1992; Barzetti, 1993). Finalmente, Richard (2005, 2006) ha sugerido utilizar a manera de diagnóstico rápido para determinar el área de conservación mínima efectiva de un ANP, los fundamentos anteriores materializados en especies claves que se ubican en la punta de la pirámide trófica y cuya conservación (Población Viable Mínima, según Meffe et al., 1999) garantizaría la de la biodiversidad relacionada, entre otros, por mecanismos de regulación de tipo top down (Achá \& Fontúrbel, 2005). Este efecto paraguas o "dominó”, ha quedado manifiesto en experiencias en ANP de situaciones pre y post extinción de especies claves de félidos y/o cánidos. De ellas, tal vez la mejor documentada es la erradicación del lobo (Canis lupus lupus) en el Parque
Nacional Yellowstone (de acuerdo a Smith et al., 2003) y las consecuencias ecosistémicas derivadas.

Para Bolivia, no se cuenta con estudios al respecto y hemos querido aplicar los conceptos descriptos al Parque Nacional Torotoro (PNTT) del Sistema Nacional de Áreas Naturales Protegidas (SNAP), administrado por el Servicio Nacional de Áreas Protegidas de Bolivia (SERNAP). Consecuentemente, los objetivos de la presente investigación fueron los siguientes:

- Analizar la relación perímetro-superficie actual del Parque Nacional Torotoro.

- Determinar, a partir del método de especies claves (Richard, 2005, 2006), la superficie mínima e indispensable para justificar los objetivos de conservación oficiales (SERNAP) del Parque Nacional Torotoro.

- Contrastar la superficie obtenida a partir del método de especies claves con la superficie actualmente asignada del PNTT.

- Proponer alternativas para rediseño del PNTT acorde resultados obtenidos de los objetivos anteriores.

\section{Materiales y métodos}

Área de estudio

El Parque Nacional Torotoro (Figura 1) se encuentra en la región norte del Departamento de Potosí (Bolivia). Está íntegramente incluido dentro del Municipio de Torotoro de la provincia Charcas. Los límites del mismo son: 6553'00', - 6544'00',

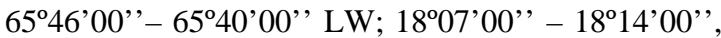
$18^{\circ} 01^{\prime} 00^{\prime \prime}$ - 1809'00', LS. El área, tiene una superficie asignada oficialmente (SERNAP 2000) de 16.570 ha.

El PNTT se ubica en el área de la Cuenca de los ríos Caine - Grande, que a su vez son afluentes de la cuenca del Amazonas. Geológicamente. Comprende elementos bien definidos del Paleozoico y del Mesozoico (Torrico \& Lara, 2000). De acuerdo a la clasificación de Navarro \& Maldonado (2002), se encuentra comprendido en la región biogeográfica andina, e incluye ecosistemas correspondientes a dos provincias y tres distritos biogeográficos a saber: Provincia biogeográfica de la Puna Peruana (Distritos biogeográfico del Tunari y biogeográfico de montañas interandinas) y Provincia Boliviano-Tucumana (Distrito biogeográfico del Caine). Los objetivos de creación del área fueron "conservar el patrimonio biológico, geológico, espeleológico y arqueológico del área” (sic. Becerra et al., 1989). De acuerdo con Torrico \& Lara (2000), el PNTT abarca tres zonas de vida (bosque seco templado, monte espinoso templado, y bosque húmedo montano subtropical), $\mathrm{y}$ comprende cuatro pisos altitudinales (termoandino, mesoandino, supraandino, y oroandino). 


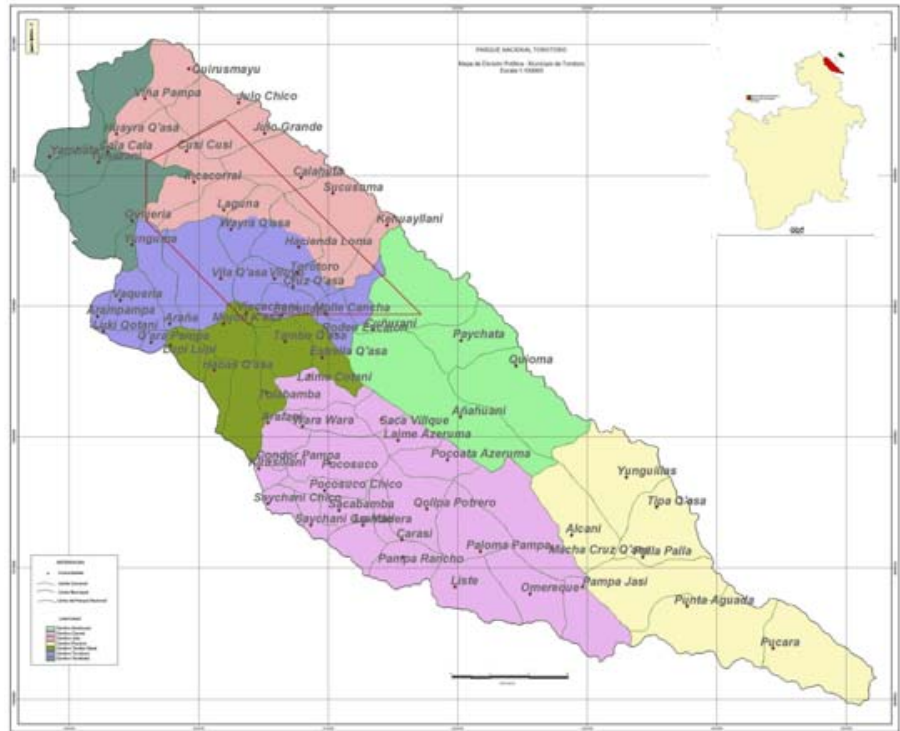

Figura 1: Mapa político de la Provincia Charcas del Departamento de Potosí (Bolivia), donde se encuentra el Parque Nacional Torotoro, delimitado por la línea roja (Modificado de García-Crispieri et al., 2006a).

\section{Diseño experimental}

Análisis de la relación perímetro-superficie actual del Parque: Se aplicó el método de relación superficie perímetro y que considera el efecto de borde (Meffe et al., 1999).

Determinación de especies clave y contrastación de superficie: Acorde con Richard (2005, 2006), las especies más indicadas para tal efecto son aquéllas que presentan mayor área de campeo en el parque. En tal sentido y, como se indicara en trabajos previos (Richard, 2006), dichas especies para ecosistemas terrestres son, entre los mamíferos, los félidos y cánidos y, entre las aves, los Falconidae, Cathartidae y Accipitridae, principalmente. Para tales especies se necesita conocer dos variables:

1) El área de campeo en el ambiente tratado. Este dato, es fundamental aproximarlo lo más posible al/los ambiente/s que se encuentran en el ANP, dado que se trata de un parámetro variable para una misma especie acorde a la capacidad de carga (y por tanto, biodiversidad) del ecosistema. A modo de ejemplo, el área de campeo de un jaguar (Panthera onca) en el área chaqueña (ambiente semiárido) será mayor que el correspondiente a Amazonía (ambiente húmedo y biodiverso) (Navarro \& Maldonado, 2002).

En este sentido, se han aproximado una serie de factores de corrección para la capacidad de carga de las tres ecoregiones presentes en el PNTT y las zonas de transición respectivas. Para ello se realizó un cálculo empírico (García-Crispieri et al., 2006 a) basado en la altitud y los bioclimas y tomando como referencia los datos de Navarro \& Maldonado (2002) para cada ecoregión así como la influencia de los factores de altitud y bioclima (It, según descripción y con valores de Navarro \& Maldonado, 2002; Pestalozzi \& Torrez, 1998), de la siguiente manera:

Factor de corrección $=\frac{\text { (altitud media }-2100) *(730-I t)}{100000}$

Dicha fórmula se incluye únicamente para fines explicativos en el presente trabajo, por lo que no amerita mayor explicación de la misma en este documento.

2) Número $\mathrm{M} \quad \mathrm{o}$ Población Viable Mínima (PVM) (Meffe et al., 1999). Este parámetro está referido al número mínimo de individuos que se necesitan para la perpetuación de la especie. Se considera para este número la diversidad genética intrínseca necesaria para la conservación de la población en el tiempo, evitando fenómenos derivados de endogamia, erosión genética, etc. Dado que no se conoce la PVM de todas las especies, con frecuencia se utiliza el de las especies conocidas.

Con los datos estipulados se calcula la superficie mínima e indispensable para que el área en estudio cumpla con los objetivos conservacionistas prefijados, según la siguiente fórmula (Richard, 2006):

$$
\mathrm{SMI}=\mathrm{H} \times \mathrm{M}
$$

Donde:

SMI = Superficie mínima e indispensable para justificar los objetivos del ANP.

$\mathrm{H}$ = Área de campeo del predador con mayor necesidad de superficie registrado para el ANP.

M (o PVM) = Número mínimo de individuos de dicha especie, necesario para su perpetuación.

Cabe destacar que, en todos los casos, los SMI calculados se basan en los valores mínimos obtenidos de estudios previos y partiendo del supuesto de una uniformidad de hábitats que en realidad, no existe en el PNTT. Consecuentemente, los valores SMI calculados en el presente trabajo, deben ser considerados mínimos.

En función de lo anteriormente expuesto y para el caso de estudio (PNTT), se tomó como referencia dos especies de felinos y un cánido. Entre las primeras, se consideró al puma (Puma concolor), felino de mayor tamaño del PNTT, que cumple con el perfil de especie clave para el cálculo de superficie mínima del ANP y que cuenta con mayor número de estudios sobre área de campeo y PVM en Latinoamérica (Currier, 1983; Bulin et al., 2004). Complementariamente, se tomó el gato andino (Oreailurus jacobita) por las necesidades de conservación del mismo en todo su rango de distribución. En cuanto al cánido, se consideró al único representante silvestre de la familia en el PNTT, el zorro (Lycalopex culpaeus). Este estudio se basa en 
las listas y distribución de fauna (en especial de las especies aquí tratadas) relevadas en campo por García-Crispieri et al. (2006 a).

Para el cumplimiento del último objetivo se tomó en cuenta los resultados emergentes de los puntos precedentes.

\section{Resultados y discusión}

Análisis perímetro - superficie

Acorde con los fundamentos de las relaciones para perímetro vs. superficie ideales, el diseño actual del PNTT corresponde a la figura geométrica de un paralelepípedo con un perímetro mayor debido a algunas irregularidades en sus vértices (ver la Figura 1), partiendo de la base de que las ANP circulares minimizan las distancias de dispersión en la misma, favoreciendo el restablecimiento de poblaciones que están localmente extintas en el ANP. Como contraparte, los diseños alargados (como el caso del PNTT) de la misma superficie que uno circular puede ocupar varios hábitats heterogéneos $\mathrm{y}$ por tanto favorecen la fragmentación e insularidad (y por tanto, a la extinción) de los mismos dentro del área. Sin embargo, en aquellos casos en los que la inmigración puede ser más importante que la extinción, un ANP alargada podría ser la mejor opción, de acuerdo a un fenómeno de compensación de ejes (la relación natalidad-mortalidad se compensa con la relación emigración-inmigración) y siguiendo los criterios de Thomas \& Kunin (1999). En el caso del PNTT, la relación perímetro - superficie calculada es de 345.29 metros de perímetro por cada $\mathrm{km}^{2}$ de superficie.

Superficie mínima indispensable

Puma concolor: Para el puma (Ferrari et al., 1984; Hansen, 1992; Parera, 2002; Currier, 1983), se tomó en consideración los siguientes datos: la densidad mínima promedio de pumas en hábitats de tipo chaqueño (situación de mayor biodiversidad del PNTT y de su zona de amortiguamiento (Figura 2) en Bolivia. La misma ha sido calculada (Cuéllar \& Noss 2003) en $30 \mathrm{~km}^{2}$ para hembras y $50 \mathrm{~km}^{2}$ para machos. Cabe destacar que, el territorio de un macho puede incluir al de dos hembras (Currier 1983, Cuéllar \& Noss 2003). Estos datos indican que la densidad de pumas, en este tipo de hábitat, es de un adulto cada 25 $\mathrm{km}^{2}$. En el Chaco de la llanura aluvial de la región de la Chiquitanía boliviana (Cuéllar \& Noss 2003) la

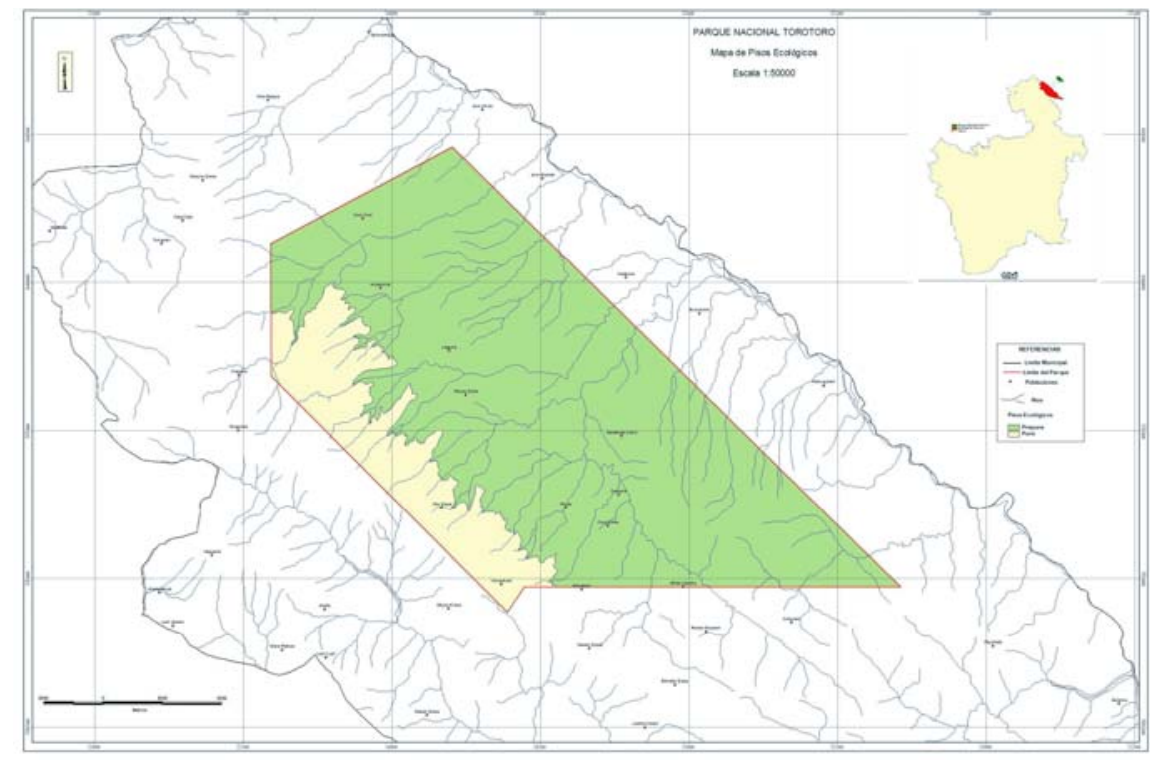

Figura 2: Mapa de ecoregiones del Parque Nacional Torotoro (Modificado de García-Crispieri et al. 2006a).

densidad es mayor, alcanzando un adulto cada $15 \mathrm{~km}^{2}$ (Cuéllar \& Noss, 2003). En tanto que la PVM mínima oscilaría (Ledezma et al., 2004; Bulin et al., 2004) entre los 550 a 7000 individuos. Luego y acorde con la fórmula propuesta por Richard $(2005,2006)$ y tomando los valores de PVM y de área de campeo mínimos para la especie se tiene que:

SMI ${ }_{\text {Puma concolor }}=15\left(\mathrm{~km}^{2}\right) \times 550$ (i) $=8.250 \mathrm{~km}^{2}$ (825.000 ha)

Cabe destacar que si bien se tomó como área de campeo, el menor valor conocido para Bolivia, este corresponde al área de Chaco (Cuéllar \& Noss, 2003) que se extiende en la zona externa de amortiguamiento hasta los márgenes del río Caine (Figura 3). 


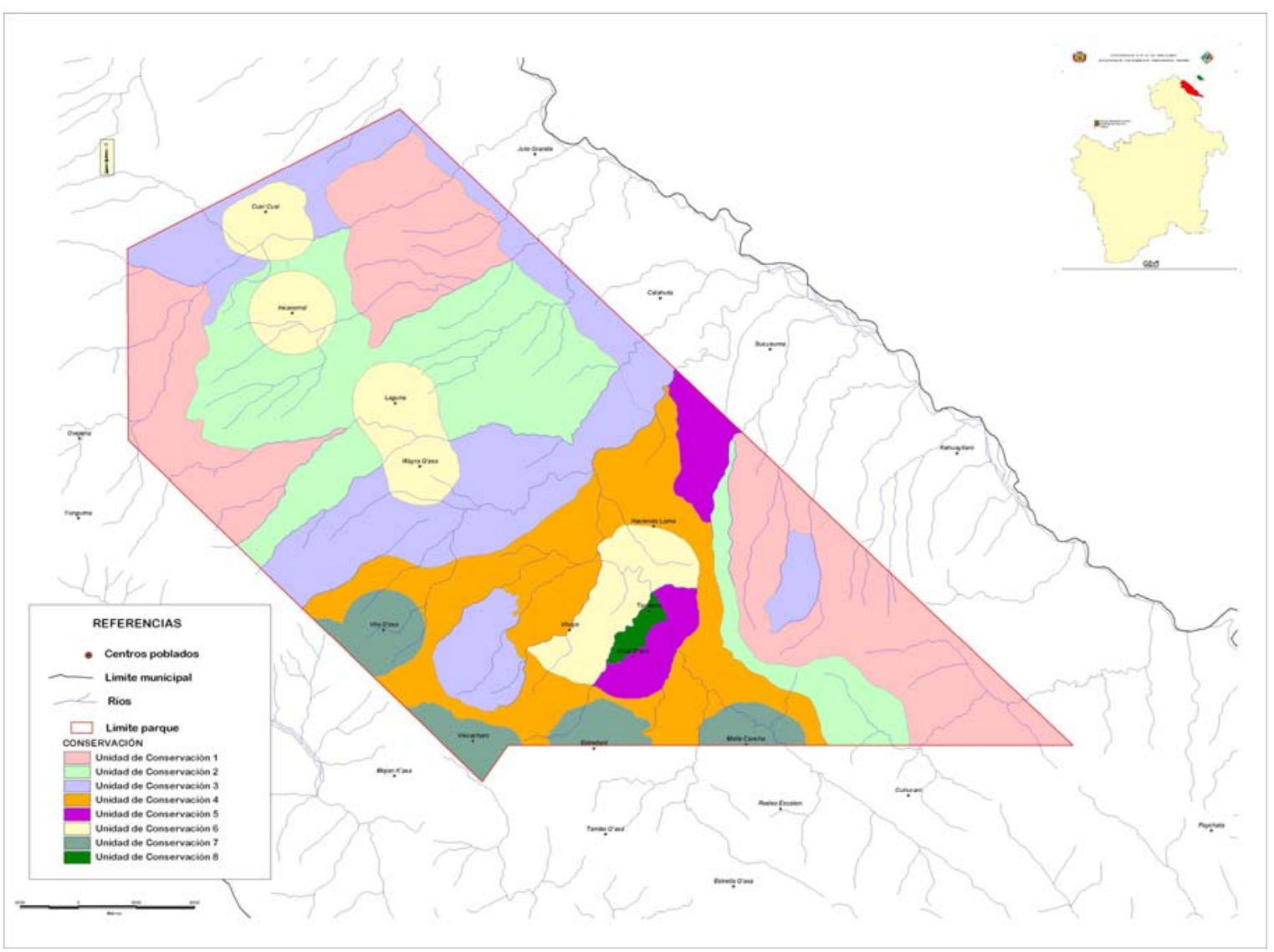

Figura 3: Mapa de estado de conservación del Parque Nacional Torotoro. La Unidad de Conservación 1 corresponde a sitios prístinos, 2 a muy buenos, 3 a buenos, 4 a regulares, 5 a deficientes, 6 a altamente intervenidos, 7 a altamente modificados y 8 a sitios artificiales y/o construidos (Modificado de GarcíaCrispieri et al., 2006 a).

Esta región, corresponde al área de mayor biodiversidad del PNTT pero su representatividad relativa es sólo cercana al $10 \%$ de la superficie actual del parque. El restante 90 \% corresponde a pisos más elevados (17\% para la ecoregión de puna y $83 \%$ para la ecoregión de prepuna) y formaciones con una biodiversidad y por tanto capacidad de carga, mucho menor. Consecuentemente, los valores SMI para las restantes áreas del parque (90\% de superficie), tenderán a ser mucho más elevados al aquí obtenido. En este sentido, para esta últimas áreas, se debe aplicar el factor de corrección calculado para las mismas (Tabla 1), respecto al área chaqueña y variables ambientales, debiendo multiplicarse los valores de superficie calculados, por los factores de corrección aproximados. Consecuentemente, en las zonas de prepuna y puna, el requerimiento de espacio sería mayor aun.
Tabla 1: Factores de corrección aproximados a aplicar en las ecoregiones prepuna, puna y zonas de transición, respecto a la ecoregión chaqueña y en función al análisis de pisos altitudinales y los bioclimas (fide García-Crispieri et al., 2006 a). Los valores de superficie para la ecoregión chaqueña se deben multiplicar por los coeficientes de la cuarta columna.

\begin{tabular}{cccc}
\hline Ecoregión & Piso altitudinal & Bioclima & $\begin{array}{c}\text { Factor de } \\
\text { corrección } \\
\text { aprox. }\end{array}$ \\
\hline Chaqueña & Termoandino & Pluvial & 1.0 \\
Transición & Mesoandino & Pluviestacional & 1.1 \\
Prepuna & Supraandino bajo & Pluviestacional & 1.2 \\
Transición & Supraandino alto & Pluviestacional & 1.3 \\
Puna & Oroandino & Xérico & $1.4-1.6$ \\
\hline
\end{tabular}

En este punto, debe tenerse en cuenta que si bien el puma es, dentro de los felinos americanos, una de las especies más eurioicas (más tendiente a r, de acuerdo con Pianka 1982) y por tanto con distribución geográfica y ambiental más amplia; dicho argumento 
no la hace menos prescindible a la hora de considerar prioridades de conservación. Como se indicó en párrafos precedentes, la falta de especies claves de los extremos de pirámides y/o redes tróficas derivan en alteraciones generales como ha podido demostrarse recientemente en el PN Yellowstone con la desaparición del lobo (Canis lupus lupus) primero y la recuperación ecosistémica consecuente con restauración del mismo en tiempos recientes (Smith et al., 2003).

En el caso de Bolivia, revisiones recientes (Flores \& Miranda, 2003) le otorgan a la variedad andina de puma (Puma concolor osgoodi), la existente en el PNTT (García-Crispieri et al., 2006 a), el estatus C2a de la UICN. Es decir, se trata de una población estimada en número menores a 2500 individuos maduros y en declinación continua observada, proyectada o inferida, en el número de individuos maduros con una estructura de la población severamente fragmentada (por ejemplo, cuando se estima que ninguna población contiene más de 250 individuos maduros). Cabe señalar que la revisión del estatus de conservación de Flores \& Miranda (2003) no incluye al PNTT como área de protección del puma a pesar de que su presencia está confirmada en la zona (García-Crispieri et al., 2006 a).

Oreailurus jacobita: De manera similar a la especie anterior, el gato andino está catalogado como En Peligro C2a por la UICN, lo cual significa que el tamaño poblacional estimado es de menos de 2500 individuos maduros, con una tendencia a disminuir y ausencia de subpoblaciones que contengan más de 250 individuos maduros (Villalba et al., 2005; Flores \& Miranda, 2003; Nowell \& Jackson, 1996). Para dicha especie, la causa principal de declinación indicada recientemente (Villalba et al., 2005) es la pérdida, fragmentación y modificación antrópica de su hábitat. Curiosamente y a pesar de ser una especie de existencia verificada en el interior del PNTT (GarcíaCrispieri et al., 2006 a), la misma no figura como parte de la fauna del PNTT en publicaciones de revisión específicas recientes (Villalba et al., 2005).

Todo ello la convierte en una especie que debería considerarse como de conservación prioritaria (medido en esfuerzos de conservación) por el Plan de Manejo del PNTT. En este caso, no existe tanta información disponible como en el caso del puma, respecto a la densidad o área de campeo (Scrocchi \& Halloy, 1986; Shorter, 2006). De ellas, la más precisa es la segunda, que indica como área de campeo entre $38,8 \mathrm{~km}^{2}$ y $77.7 \mathrm{Km}^{2}$. En tanto que la PVM mínima se asume, como en el caso anterior, entre los 550 a 7000 individuos (Ledezma et al., 2004 y Bulin et al., 2004). Luego y acorde con la fórmula de Richard (2005, 2006) tomando el valor de PVM y de área de campeo mínimos para la especie se obtiene:

$$
\text { SMI Oreailurus jacobita }=38,8\left(\mathrm{~km}^{2}\right) \times 550 \text { (i) }=21.340
$$
$\mathrm{km}^{2}$ (2.134.000 ha)
Lycalopex culpaeus: Para este cánido, se cuenta con gran cantidad de información ecobiológica y genética y como el caso del puma, estamos frente a una especie eurioica (Pianka, 1982) entre los Canidae sudamericanos. Consecuentemente, posee una amplia distribución geográfica y ambiental pero, como en el caso del puma, en función de su posición trófica, su eventual desaparición derivaría en alteraciones generales de todo el ecosistema y en relativamente corto tiempo (Novaro, 1997; Cuéllar \& Noss, 2003).

Cabe destacar que carnívoros como L. culpaeus, como las de $P$. concolor y $O$. jacobita, son especies responsables de mecanismos de control de tipo topdown (Hairston et al., 1960; Power, 1992; Dile, 1992; Achá \& Fontúrbel, 2005) en el que el nivel superior de la pirámide trófica se encarga de controlar la diversidad del resto de los niveles de la comunidad, por medio de una depredación “inteligente” y selectiva (Carpenter \& Kitchell, 1987; Power et al., 1992).

Si bien no se cuenta con información del área de campo de la especie para Bolivia, se sabe que la misma varía entre 0,72 individuos $/ \mathrm{km}^{2}$ (Provincia de Neuquén, Argentina; según Novaro 1997) y 1,3 individuos $/ \mathrm{km}^{2}$ (Parque Nacional Torres del Paine, Chile; Novaro 1997). La PVM mínima oscilaría (Ledezma et al. 2004) entre los 550 a 7000 individuos. Con lo que aplicando la fórmula de Richard (2005, 2006) tomando los valores mínimos de PVM y de área de campeo para la especie, se obtiene:

SMI Lycalopex culpaeus $=0,72\left(\mathrm{~km}^{2}\right) \times 550$ (i) $=396$ $\mathrm{km}^{2}$ (39.600 ha)

Contrastación con la superficie actual del PNTT

Oficialmente (según SERNAP 2000), el PNTT, en sus límites actuales, posee una superficie de 16.570 hectáreas (aproximadamente $166 \mathrm{~km}^{2}$ ). Con lo que, a la fecha, se constituye en el ANP más pequeña de Bolivia. Desde esta dimensión, surge claramente y en forma en extremo significativa que dicha superficie, como parche o isla, limitada incluso por los cauces de los ríos Caine, Torotoro, 7UP y San Pedro, no sería adecuada para sostener Poblaciones Viables Mínimas de las especies claves aquí testadas y probablemente las de otros grupos también (otros carnívoros, rapaces, etc.).

Efectivamente, la especie que más territorio necesitaría es Oreailurus jacobita con una SMI de $21.340 \mathrm{~km}^{2}$ (unas 128 veces la superficie del PNTT) y la que poseería menos requerimientos es Lycalopex culpaeus con $396 \mathrm{~km}^{2}$ (unas 2 veces y media la superficie del PNTT).

Hay que destacar que, al interior del PNTT, existen 11 centros poblados concentrados principalmente en la zona central del área, creando una estructura clásica de parches con las zonas de menor intervención (Figura 3). En consideración a esta situación, se evidencia que apenas el 47\% del área del PNTT (unas 7730 ha) tiene un estado de conservación e intervención aceptables para las 
poblaciones de predadores analizados. El resto del área (53\%), se encuentra intervenida, poblada y con barreras antrópicas que dificultan la vinculación entre los parches y la complementación del paisaje (en el sentido que describen Shealagh et al., 2000).

Todo ello nos lleva a suponer que la insularidad creciente que viene desarrollando el PNTT por la intervención humana (García-Crispieri, 2006 a) incidirá en el corto plazo en la extinción (Carpenter, 2002) de las especies con el perfil aquí utilizado derivando en un desequilibrio ecológico de proporciones ahora difícil de calcular en función del alto grado de intervención humana descontrolada dentro del área (García-Crispieri et al., 2006 a).

Los resultados del presente nos inducen a reflexionar sobre la importancia de analizar, como parte del diseño de ANP, el tema de la conservación a nivel del ecosistema (Fontúrbel, 2004a, 2004b, 2004c) para garantizar la persistencia a largo plazo de las poblaciones (Schwartz et al., 2000; Hector et al., 2001) del área de estudio y otras similares, y no limitarlos a esfuerzos de conservación a corto y/o mediano plazo.

\section{Conclusiones y recomendaciones}

El uso de especies clave, en la regulación de la biodiversidad ecosistémica, con requerimientos amplios de área de campeo y PVM elevados se muestra como un método idóneo para evaluaciones rápidas de objetivos de conservación integrales de áreas naturales protegidas al actuar como un efecto paraguas sobre las demás especies que poseen requerimientos menores. Asimismo, se constituye en un método rápido y confiable para el cálculo de la superficie mínima y forma que las mismas deberían tener en función de los objetivos de conservación y por tanto, para el diseño de ANP.

Los análisis de la relación superficie-perímetro y SMI para $P$. concolor, O. jacobita y L. culpaeus, señalan que el diseño actual del PNTT no cumple con los objetivos oficiales (SERNAP) de conservación de la biodiversidad asignados; ya que la superficie y la forma del mismo no permiten sostener a las poblaciones de especies clave para el control de la diversidad de la comunidad.

Los resultados del trabajo indican que el PNTT, actualmente no cumple con los objetivos de conservación del SERNAP (Pabón et al., 2002) para la categoría de manejo de Parque Nacional. Por ello se debería replantear su categoría de manejo a una más pertinente. Un plan de mitigación deberá plantear una ampliación legal del área del PNTT y/o la modificación de sus límites (en evidencia que los límites artificiales actuales producen un elevado efecto de borde y la insularidad del ANP). García -Crispieri et al. (2006 b) plantearon como propuesta innovadora, el concepto de Municipio-Área Protegida como modelo a seguir acorde al contexto de la problemática actualmente emergente en Bolivia, de ANP de reducida superficie. Bajo este modelo la superficie del PNTT, sumada a la del Parque Departamental Jucumarini (PDJ) (Miranda 2005) y la zona externa de amortiguamiento (ZEA) del primero, ocuparían la mayor parte de la superficie del Municipio de Torotoro (92 \%, equivalente aproximado a $1.079 \mathrm{~km}^{2}$ ) y la administración conjunta (PNTT+ZEA+PDJ, ver Figura 4), desde el Municipio, permitirá generar un espacio geográfico continuo de mayor extensión que conduzca a cumplir con los objetivos de conservación de ambas áreas protegidas y de la respectiva ZEA.

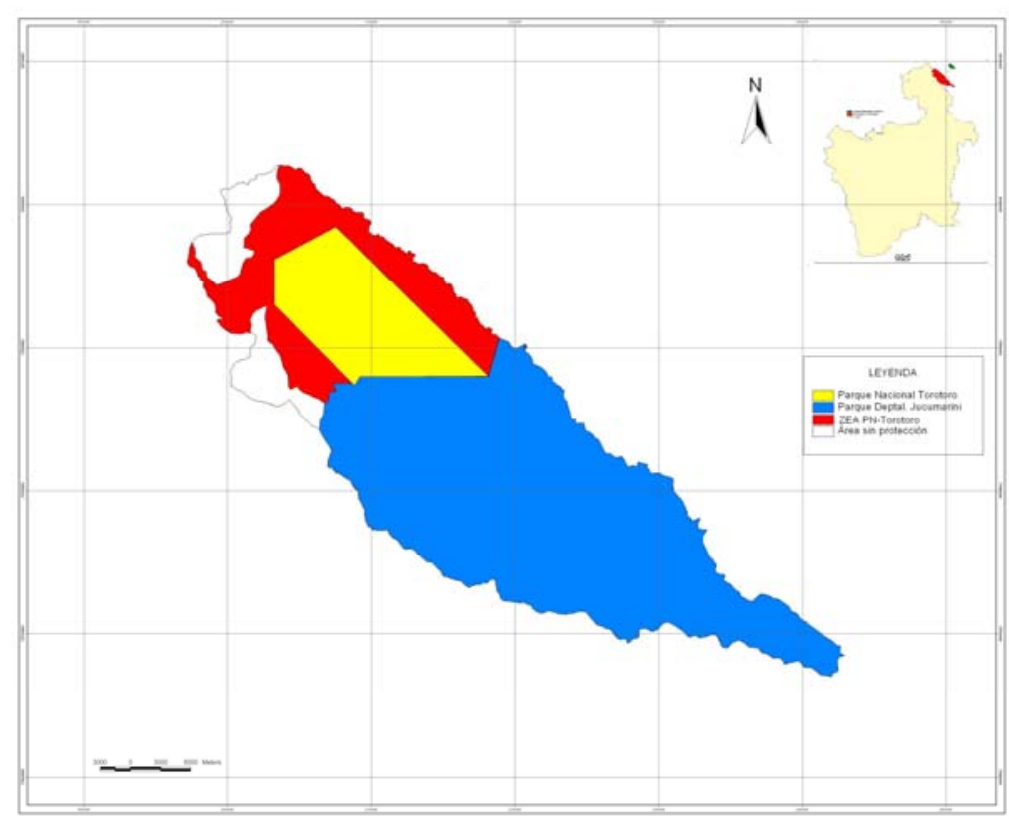

Figura 4: Mapa de ubicación del Parque Nacional Torotoro, su zona externa de amortiguamiento, y el Parque Departamental Jucumarini, en el Municipio de Torotoro Elaboración y SIG: Francisco Fontúrbel, Sergio Fernández).
Sin embargo, no se debe perder de vista el tema de la aplicación del factor de corrección de acuerdo a las distintas ecoregiones (ver Tabla 1) y al tema de la intervención humana; ya que en el municipio de Torotoro existen más de 30 centros poblados y, de acuerdo a lo que plantea Carpenter (2002), el pensar en una ecología de largo plazo representa considerar también el factor humano, como parte integral e indivisible del ecosistema.

Finalmente, se debe considerar la conectividad del área propuesta (PNTT+ZEA+PDJ) a través de 
corredores de biodiversidad (Rabinowitz, 2003) como contribución a la mitigación del problema de insularidad descrito. En este sentido, actualmente, el PNTT forma parte de la zona de amortiguamiento del Corredor Vilcambamba-Amboró (García-Crispieri et al., 2006 a, 2006 b, 2006 c), aunque la relación efectiva que tiene con el mismo, es cuestionada, principalmente por un tema de continuidad geográfica (barreras físicas). Consecuentemente y sin desechar lo anterior, sería más relevante, para este caso, el pensar en un corredor de menor escala que incorpore al conjunto PNTT+ZEA+PDJ en un corredor triple junto con el Área Natural de Manejo Integrado El Palmar y el Parque Nacional y Área Natural de Manejo Integrado Serranía del Iñao (SERNAP) y con una continuidad más consistente de formaciones de vegetación y elementos faunísticos (Beck, 1985; Pestalozzi \& Torrez, 1999; Navarro \& Maldonado, 2002; Ribera, 2003), como se nuestra en la Figura 5.

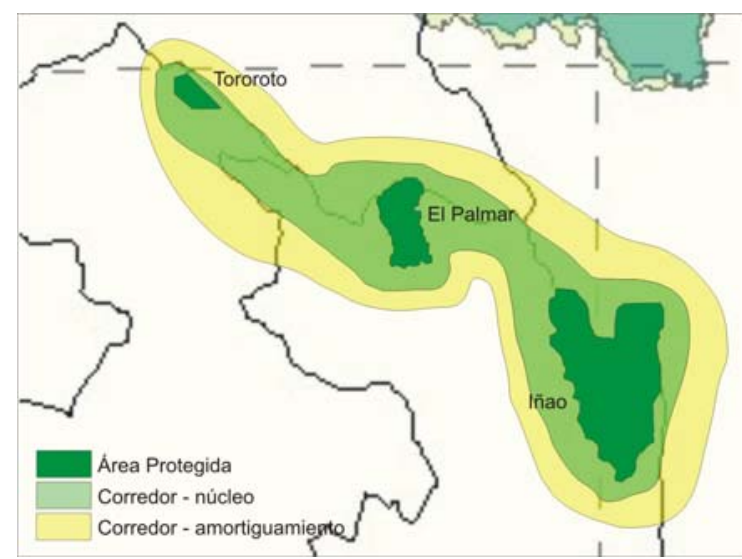

Figura 5: Localización aproximada (no está a escala) del corredor propuesto Torotoro-El Palmar-Serranía del Iñao (Según propuesta de Garcia-Crispieri et al. 2006 a).

\section{Agradecimientos}

A Sergio Fernández por la elaboración de la cartografía base y la gestión de Sistemas de Información Geográfica. A Darío Achá por los comentarios al manuscrito.

\section{Literatura citada}

Achá D. \& Fontúrbel F. 2005. Modelos de control de la diversidad en la comunidad: top-down, bottom-up o ambos. En: Fontúrbel, F. (ed.). Hábitat: El Lugar Donde Vivimos. Maestría en Recursos Naturales y Gestión Ambiental, Escuela Militar de Ingeniería. CD-ROM interactivo, Escuela Militar de Ingeniería y Ed. Publicaciones Integrales, La Paz.

Acosta-Jamett G., Simonetti J.A., Bustamante R.O. \& NDunstone N. 2003. Metapopulation approach to asses survival of Oncifelis guigna in fragmented forests of central Chile: a theoretical model. J. Neotrop Mammal $.10(2): 217-229$.
Barzetti V. (ed.). 1993. Parques y progreso: Áreas protegidas y desarrollo económico en América Latina y el Caribe. UICN, BID. xiv + 258 p. USA.

Becerra R., Guyot J.L. \& Salazar D. 1989. Estudio para la creación del Parque Nacional Torotoro. Publicación de la Asociación Conservacionista de Torotoro, La Paz.

Beck S.G. 1985. Flórula ecológica de Bolivia. Ecología en Bolivia. 6: 1-41.

Bulin B., Fullington S. \& Meinerz R. 2004. Population viability analysis of the mountain lion (Puma concolor) in the State of Wisconsin. Felids of Wisconsin, workpaper [online].

Carpenter S.R. \& Kitchell J.F. 1987. Consumer control of lake productivity. BioScience. 38: 764-769.

Carpenter S. 2002. Ecological futures: building an ecology for the long now. Ecology. 83: 2069-2083.

Cuéllar E. \& Noss A. 2003. Mamíferos del chaco y la chiquitanía de Santa Cruz, Bolivia. Ed. FAN, Santa Cruz, Bolivia.

Currier J.P. 1983. Felis concolor. American Society of Mammalogists. Mammalian Species. 200: 1-7.

Diamond J.M. 1975. The island dilemma: lessons of modern biogeographic studies for the design of natural reserves. Biol. Conserv. 7: 129-146.

Diehl S. 1992. Fish predation and benthic community structure: the role of omnivory and habitat complexity. Ecology. 73(5): 1646-1661.

Fernández H.R. 2000. Zoogeografía aplicada: Conservación de la biodiversidad. FCN e IML Serie Monográfica y didáctica $\mathrm{n}^{0}$ 16: 19 p. Argentina.

Ferrari M., Reig O., Saltzman J., Palermo M.A. \& Marchetti B. 1984. Los gatos silvestres. Fauna Argentina .57.

Flores E. \& Miranda C. 2003. Fauna amenazada de Bolivia ¿Animales sin futuro?. Ministerio de Desarrollo Sostenible, Bolivia, UICN.

Fontúrbel F. 2004 a. Manejo y conservación de fauna silvestre: un enfoque conceptual, metodológico y práctico para el tercer milenio. CD-ROM interactivo. Escuela Militar de Ingeniería y Ed. Publicaciones Integrales, La Paz.

Fontúrbel F. 2004 b. Conservación de ecosistemas: un nuevo paradigma en la conservación de la biodiversidad. Ciencia Abierta Internacional. 23: 18.

Fontúrbel F. 2004 c. Un ejemplo de la necesidad de la conservación de ecosistemas: la eutrofización localizada en el lago Titikaka. Revista de de Biología.org 15.

García-Crispieri G., Fontúrbel F., Richard E., Brun N., Burgoa N., García M. \& Fernández S. 2006 a. Actualización del Plan de Manejo del Parque Nacional Torotoro. Gregory L. Morris Engineering - GEF II/SERNAP - EcoDreams, documento de trabajo, La Paz.

García-Crispieri G., Fontúrbel F. \& Richard E. 2006 b. Innovación en gestión y conservación: el concepto de Municipio-Área Protegida. Ed. EcoDreams Publicaciones Integrales, La Paz.

García-Crispieri G., Fontúrbel F. \& Richard E. 2006 c. Propuesta para un desarrollo económico local auténtico para Bolivia: la creación de un municipio área natural protegida y ecomuseo; el Parque Nacional Torotoro (Potosí, Bolivia) como ejemplo. In Vestigium. 1(3): 7 19. 
Forman R. \& Godron M. 1986. Landscape Ecology. J. Wiley y Sons.

Hairston N.G., Smith F.E. \& Slobodkin L.B. 1960. Community structure, population control and competition. Am. Natur. 94: 421-425.

Hansen K. 1992. Cougar: the American lion. Flagstaff: Mountain Lion Foundation and Northland Press.

Harrison S. 1991. Local extinction in a metapopulation context: an empirical evaluation. Biological Journal of the Linnean Society. 43: 3-16.

Hastings A. \& Harrison S. 1994. Metapopulation dynamics and genetics. Annu. Rev. Ecol. Syst. 25: 167-188.

Hector A., Joshi J., Lawler S.P., Spehn E.M. \& Wilby A. 2001. Conservation implications of the link between biodiversity and ecosystem functioning. Oecologia. 129: 624-628.

Klug W. \& Cummings M. 1999. Conceptos de genética. $5^{\circ}$ ed., Prentice Hall, Madrid.

Ledezma J.C., Painter L. \& R. Wallace. 2004. Identificación de vacíos de conservación y áreas posibles para conservación de poblaciones mínimas viables de especies con amplios requerimientos espaciales. WCS, documento de trabajo, La Paz.

Meffe G., Caroll C.R. et al. 1999. Principles of conservation biology. 2nd Ed. Sinauer.

Miranda C. 2005. Identificación de potenciales áreas protegidas en el Departamento de Potosí. PCDSMA Savia, La Paz.: 73-81.

Moyle P.B. \& Sato G.M. 1990. On the design of preserves to protect native fishes. En: Battle against extinction: Native Fish Management in the American West: 155284. W.L. Minckley and J. E. Deacon (Eds). University of Arizona Press, Tucson.

Navarro G. \& Maldonado M. 2002. Geografía ecológica de Bolivia: Vegetación y ambientes acuáticos. Fundación Simón I. Patiño, Cochabamba.

Novaro A.J. 1997. Pseudalopex culpaeus. American Society of Mammalogists. Mammalian Species. 558: 1-8 .

Nowell K \& Jackson P. (eds.). 1996. Wild Cats: Status Survey And Conservation Action Plan UICN, Gland, Switzerland.

Pabón L., Peres J.A. \& Czerwenka C. 2002. Guía para la elaboración de planes de manejo para áreas protegidas de Bolivia. SERNAP-GTZ-MAPZA, La Paz.

Parera A. 2002. Los mamíferos de la Argentina y la región austral de Sudamérica. Editorial El Ateneo.

Pestalozzi H.U. \& Torrez M.A. 1999. Flora ilustrada altoandina. Herbario Nacional de Bolivia - Herbario Forestal Nacional "Martín Cárdenas", Cochabamba.

Pianka E.R. 1982. Ecología Evolutiva. Ediciones Omega, S.A., Casanova, 220, Barcelona - 36. X + 365 p.

Power M.E. 1992. Top-down and bottom-up forces in food-webs: do plants have primacy?. Ecology. 73(2): 733-746.

Power M.E., Marks J. \& Parker M. 1992. Variation in the vulnerability of prey to different predators: communitylevel consequences. Ecology. 73: 2218-2223.

Rabinowitz A. 2003. Manual de capacitación para la investigación de campo y la conservación de la vida silvestre. Editorial FAN, Santa Cruz.

Ribera M.O. 2003. El Sistema Nacional de Áreas Protegidas (SNAP). pp 358-379. En: Ibisch, P. \& G. Mérida (eds.). Biodiversidad: La Riqueza De Bolivia, Estado De
Conocimiento Y Conservación. Editorial FAN, Santa Cruz.

Richard E. 2005. Contrastación del grado de confiabilidad del diseño de áreas naturales protegidas en relación a especies $\mathrm{K}$ de la cima de pirámides tróficas incluidas en las mismas. Bolivia. En: E. Richard (Ed.), Ecología para Ingeniería Ambiental: Syllabus, Guía de trabajos prácticos, Work Papers y materiales de estudio y consulta ( $1^{\circ}$ Edición). CD ROM interactivo. Editorial EcoDreams Multimedia y Universidad de Aquino de Bolivia. La Paz, Bolivia.

Richard E. 2006. Paradigmas en el diseño de áreas naturales protegidas con especial referencia a los problemas de insularidad. En: En: E. Richard (Ed.), Ecología para Ingeniería Ambiental: Syllabus, Guía de trabajos prácticos, Work Papers y materiales de estudio y consulta ( $1^{\circ}$ Edición). CD ROM interactivo. Editorial EcoDreams Multimedia y Universidad de Aquino de Bolivia. La Paz, Bolivia.

Schoener A. 1988. Experimental island biogeography. En: Analytical Biogeography: En Analytical Biogeography: 483 - 512. A. A. Myers y P. S. Giller (Eds). Chapman y Hahh, Londres.

Schwartz M.W., Brighman C.A., Hoecksema J.D., Lyons K.G., Mills M.H. \& Mentgen P.V. 2000. Linking biodiversity to ecosystem function: implications for conservation ecology. Oecologia. 122: 297-305.

Scrocchi G.J. \& Halloy S.P. 1986. Notas sistematicas, ecologicas, etologicas y biogeográficas sobre el gato andino Felis jacobita Cornalia (Felidae, Carnivora). Acta. Zool. Lilloana. 38(2): 157-170.

SERNAP. 2000. Información técnica del Sistema Nacional de Áreas Protegidas de Bolivia. Plural editores, La Paz.: 124-129.

Shealagh E.P., Fahrig L. \& Merriam H.G. 2000. Landscape complementation and metapopulation effects on Leopard Frog populations. Ecology. 81(9): 2498-2508.

Shorter C.M. 2006. Andean mountain cat - Oreailurus jacobita. Tigerhomes, http://www.tigerhomes.org/wildcats/wc-andean-mountain-cat.cfm Consultada el 29 de marzo de 2006.

Smith D.W., Peterson R.O. \& Houston D.B. 2003. Yellowstone after wolves. International Wolf Center. www.wolf.org. Consultada el 9 de Marzo de 2006.

Smith R.L. \& Smith T.M. 2001. Ecología. $4^{\circ}$ edición, Ed. Addison-Wesley, Madrid, pp 319-322, 442-587.

Sullivan A.L. \& Shaffer M.L. 1975. Biogeography of the Megazoo. Science. 189: 13-17.

Thomas C.D. \& Kunin W.E. 1999. The spatial structure of populations. Journal of Animal Ecology. 68: 647-657.

Torrico M.E. \& Lara R. 2000. Parque Nacional Torotoro: Una guía para su interpretación. ACT, Konrad Adenauer, LIDEMA, La Paz.

Vides R. \& Korn H. 1992. Las zonas "buffer" como alternativas para el manejo de áreas silvestres protegidas en Tucumán: EL caso de la sierra de San Javier. Resúmenes II Congreso Regional El NOA y su Medio Ambiente, Salta, Consejo de Investigación de la Universidad Nacional de Salta.

Villalba L., Lucherini M., Walker S., Cossios D., Iriarte A., Sanderson J., Gallardo G., Alfaro F., Napolitano C. \& Sillero Zubiri C. 2004. El gato andino. Plan de acción para su conservación. Ed. Alianza Gato Andino. La Paz, Bolivia. 
Diciembre 2006

Institución actual y donde se realizó el trabajo:

${ }^{1}$ EcoDreams, Consultora Ecobiológica y Académica Internacional. División de Investigación. P.O. Box \# 180, La Paz, BOLIVIA. E-mail: chelonos@gmail.com

${ }^{2}$ Director del Instituto de Investigaciones y Desarrollo y Profesor Titular de Ecología Universidad de Aquino de Bolivia, La Paz, BOLIVIA.

${ }^{3}$ EcoDreams, Consultora Ecobiológica y Académica Internacional. División de Investigación. P.O. Box \# 180, La Paz, BOLIVIA. E-mail: fonturbel@gmail.com

${ }^{4}$ EcoDreams, Consultora Ecobiológica y Académica Internacional. División de Investigación. P.O. Box \# 180, La Paz, BOLIVIA. E-mail: gonigar@gmail.com

Dirección de contacto del autor para correspondencia:

* Francisco Fontúrbel R.

Casilla postal \# 180, La Paz, BOLIVIA

Tel./Fax +591-2-2423238

Correo electrónico: fonturbel@gmail.com; fonturbel@yahoo.es 